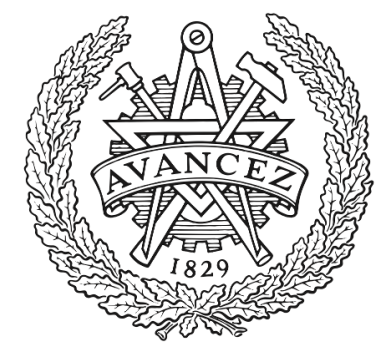

CHALMERS

UNIVERSITY OF TECHNOLOGY

\title{
From tacit knowledge to visual expertise: Eye-tracking support in maritime education and training
}

Downloaded from: https://research.chalmers.se, 2023-04-26 11:51 UTC

Citation for the original published paper (version of record):

Eklund, R., Sellberg, C., Osvalder, A. (2020). From tacit knowledge to visual expertise: Eye-tracking support in maritime education and training. Advances in Intelligent Systems and Computing, 1211: 269-275.

http://dx.doi.org/10.1007/978-3-030-50896-8_39

N.B. When citing this work, cite the original published paper. 


\title{
From Tacit Knowledge to Visual Expertise: Eye-Tracking Support in Maritime Education and Training
}

\author{
Rikard Eklund $^{1(\bowtie)}$, Charlott Sellberg ${ }^{2}$, and Anna-Lisa Osvalder ${ }^{1}$ \\ 1 Design and Human Factors, Department of Industrial and Materials Science, \\ Chalmers University of Technology, 41296 Gothenburg, Sweden \\ rikard.eklund@chalmers.se \\ 2 Department of Applied Information Technology, University of Gothenburg, \\ 41296 Gothenburg, Sweden
}

\begin{abstract}
The maritime pilot is an expert with knowledge on a specific navigational route. The maritime pilot cadet undergoes maritime education and training in classrooms, onboard vessels and in simulators. Developing visual expertise is a basic objective. Transferring knowledge from experienced maritime pilots to maritime pilot cadets is challenging since some of this knowledge is tacit. The transference is achieved by externalization and socialization processes. The objective of this pre-study was to assess eye-tracking methodology as a tool to support maritime education and training, and for transferring tacit knowledge. The study was performed in an explorative way during simulator sessions, by interviews, questionnaires and observations. The result shows that eye-tracking methodology is useful for transferring tacit knowledge in simulator settings, but not during other parts of the education and training. The results also show that situational awareness of maritime pilot instructors and maritime pilot cadets increases when utilizing eye-tracking methodology.
\end{abstract}

Keywords: Maritime education and training - Maritime pilot - Simulator · Eye-tracking $\cdot$ Visual expertise $\cdot$ Tacit knowledge

\section{Introduction}

The maritime pilot (hereafter pilot) guides vessels in order to maintain safety, protect the environment and to ensure availability of harbors. The Swedish Maritime Administration provides pilotage and assistance in the navigation to vessels within Swedish territorial waters. On average, 32.500 pilotages are conducted each year in Sweden [1]. When the pilot has boarded a vessel, the pilot will temporarily become a member of the bridge crew assisting the Master in command of the vessel with information and advice on the route to be navigated. Pilots are also able to provide effective communication with personnel on shore, such as Vessel Traffic Service Officers and crews on tugs. While the Master always remains in command on the vessel, the pilot conveys expert knowledge and may maneuver the vessel if agreed upon [1]. The working practice of pilots is by tradition still to large extents relying on the pilots' visual outlook on the maritime environment, in combination with semi-automated systems for communication, 
navigation and surveillance [2]. The Portable Pilot Unit (PPU) is carried onboard by the pilot and is a support-tool that assists the pilot in performing safe navigation of the piloted vessel. The pilots' working situation is part of a larger safety-critical sociotechnical system [3].

The pilot is required to master two skills: ship handling and having expert knowledge on the specific route in terms of area geography, infrastructure, navigation, and oceanic as well as meteorological particularities. Ship handling is generally acquired during extensive sea going experience as a Master; expert knowledge is acquired during maritime education and training (MET) provided by a Competent Pilotage Authority (CPA). In Sweden, the CPA is the Swedish Maritime Administration. The maritime pilot cadet (hereafter cadet) undergoes MET in classrooms, using scale models, simulators and supervised pilotage training on waterways during a period of 6-12 months, in combination with formal and informal interaction with senior pilots at the pilotage station. During this process, simulators offer opportunities for cadets to train and evaluate work practices and strategies in an educational setting. Using simulators, scenarios can be specifically set up and adjusted to match requirements. The simulator also allows the cadet to gain experience, partly by making errors and learn from these through evaluations of the actions taken. Scenarios can be repeated over time to present and train different actions and solutions, to illustrate potentional arising risks or to drill the cadet in basic maneuvers [4]. Sellberg [5] as well as Hontvedt and Arnseth [6] point out that a simulator does not add much per se in terms of learning; the advantage is found in how a scenario is simulated and how routine operations as well as critical situations are resolved in interaction between the participants.

\subsection{Study Aim and Objectives}

Teaching in a simulated maritime environment generates challenges for the maritime pilot instructor (hereafter instructor) to assess where the cadets keep their eye-focus. Analysis of eye-focus is a method to evaluate if attention is allocated at the right object, at the right time, and for the accurate amount of time [7]. In previous research, eyetracking technology has been used for evaluating handling of equipment on ship bridges, as well as for observing interaction, attention, vigilance and situational awareness [8]. Eye-tracking focuses on tracking the position and movement of an eye, generating data that can be assessed, analyzed and visualized in specific software programs [8].

The objective of this explorative pre-initial study was to assess if the eye-tracking methodology is a useful tool to support tacit knowledge transfer from expert to novice pilots focusing on developing visual expertise. In particular, this study investigates if and how eye-tracking data can be useful for educational purposes in simulator-based MET of pilots, by investigating the following questions; (a) explore how the Swedish Maritime Administration conducts training of pilots, and (b) evaluate if eye-tracking can improve pilot training in terms of didactics and methodologies. Through this, eye tracking is (c) evaluated in terms of its usefulness as a tool to improve transfer of tacit knowledge when cadets develop their visual expertise and skills as pilots. 


\section{Background}

There are many definitions of expertise: Karhu [9] implies that expertise is based on extensive knowledge; Gobet [10] extends the definition and defines an expert as someone who has a superior set of knowledge, expert opinion, know-how, or expertness in an area; Johnson et al. [11] define an expert as someone who is characterized by superior performance within a specific domain of activity; according to Dreyfus and Dreyfus [12] expertise is fluid, automatic behavior without any conscious control, and developing expertise also requires a significant amount of time. In the context of maritime piloting, the cadet has gained years of experience as a Master, which is a prerequisite to become a pilot. The transfer of knowledge, in order to acquire professional skills, is achieved by interacting with experienced pilots, by experiences from the actual pilotage route, as well as through simulator sessions and briefings during and after these sessions. Furthermore, reflection (in group settings or solitarily) is important to enable professional learning [6, 7].

Experts and novices use different strategies to gain knowledge. Based on experience, the novice develops improved strategies to better interact with the specific environment; strategies that over time becomes knowledge [13]. Kasarskis et al. [14] found that dwell times in experts are shorter than in novices, indicating that experts gather the required information quicker than novices. Experts and novices furthermore fixate given information differently, based on not only the available type of information but also regarding the most important information. Conducting maritime pilotage requires a high degree of visual attention, both inside as well as outside the ship bridge. As the level of automation has increased continuously, the navigation-, communication- and surveillance-system interfaces require increasing attention. A common practise on the ship bridge is to prioritize conditions permitting the outside view, with RADAR and with other electronic navigational support systems, such as ECDIS and various forms of communication/surveillance as the first choice in low visibility or darkness. The latter is also used as a support during good weather conditions. Brown [13] outlines how expertise can be divided into a theoretical or declarative part (knowing that) as well as a practical or procedural part (knowing how). The latter is related to Polyani and the topic of tacit knowledge $[15,16]$. He stated the expression "we can know more than we can tell", thus suggesting that expertise develop over time by learning how to do things. Furthermore, Von Krogh et al. [17] regard expertise as a specific category of tacit knowledge "emphasizing the uniqueness of a person's knowledge". Tacit knowledge is a valuable asset for an organization, but since it can be difficult to express, it is often hard to convey to others. Hence, establishing means to identify, collect, document and transfer tacit knowledge is important [18]. The transfer of tacit knowledge requires personal contacts, interaction, as well as closeness and trust between members in the organization. Nonaka and Takeuchi [19] suggest that socialization is the first of two ways of transferring tacit knowledge; the novice learns from an expert by imitating, observing and by training based on instructions where tacit knowledge is directly translated to explicit knowledge. The second way is when tacit knowledge is externalized; first to explicit knowledge and then it is possible for the novice to assess it from oral presentation or documentation. Methods on how tacit 
knowledge can be transferred from experienced operators in process control have been proposed by Osvalder and Colmsjö [20]. Socialization can occur in shift teams during control room operation or by individual apprenticeship in the control room, by focusing on which visual and audible cues, patterns, and rules of thumb that are used during various operation modes, especially during disturbances. Externalization of tacit knowledge can occur if the talk aloud methodology is used during the operation work. Osvalder and Colmsjö [20] state that in this environment eye-tracking could have been an additional useful tool understand on what cues the operators focus their view during operation control.

\section{Methodology and Data}

The present study was an initial exploratory pre-study. In the first step, a literature review was performed. The literature review was conducted to assess the research field related to MET and to find studies reporting on the use of the eye-tracking methodology. In total 12 databases were searched for peer-reviewed articles. Through this search, 114 documents were selected for further analyzes. In all, 48 articles were identified as relevant. In the second step, data was collected during two cadet pilot courses using observations, interviews, a focus group session including questionnaires, and eye-tracking glasses.

The evaluation of the pilot MET and its procedures were made in order to understand how the MET was performed. Observations and interviews were used during one pilot basic course and one pilot recurrent course, resulting in total of $85 \mathrm{~h}$ of observations. In total 25 participants were enrolled on a voluntary basis; 6 pilots, 12 cadets, 5 instructors and 2 administrators/technicians. All participants were employed or probationary employed by the Swedish Maritime Adminstration. The response rate for the interviews was $76 \%$.

During the pilot basic course, eye-tracking glasses were operated by four cadets in three different simulators, in three-member crew teams during one scheduled simulator session (45 min). Each crew consisted of one Master, one observer (for educational purposes) and one cadet acting as a pilot. The cadet wore the eye-tracking glasses. Furthermore, two instructors assessed the eye-tracking equipment (including glasses, monitoring screens and software) before, during and after the scheduled pilot basic as well as recurrent simulator sessions.

Based on the literature review, a questionnaire was designed containing questions on didactics, methodology, issues in general, prerequisites and limitations during the MET, as well as questions on what is consistent with being a skilled pilot. The questionnaire served two purposes: (1) to collect the views from the instructors in a focus group setting on how they conducted their MET, and (2) if eye-tracking methodology could be used as an instructor supporting tool. The focus group consisted of 5 instructors and lasted for $2.5 \mathrm{~h}$. The discussions in the focus group were audio recorded and transcribed. A thematic inductive analysis based on Braun and Clarke [21] was used where topics were examined, categorized and then clustered.

The eye-tracking equipment was also continuously assessed in general terms of operational prerequisites and limitations during the cadet MET course, the recurrent 
course as well as during other time-periods (in total for $21 \mathrm{~h}$ ) and included both cadets, pilots and instructors. Data were collected on how the eye-tracking equipment functioned from technical, organizational, physiological and psychological perspectives based on observations and notes from evolving commentaries. The observations were documented on paper, by photography and by screen shots on the monitoring screen. The observations included operational aspects on the eye-tracking equipment: range, limitations on data storage capacity, contingency operations when loss of wireless communications, limitations for wearing normal glasses, reading glasses or contact lenses when using eye-trackers, head posture and gaze techniques, physiological and psychological discomfort when wearing eye-trackers that could affect performance during simulator sessions. Discomfort was gathered in terms of elevated stress levels, the notion of being observed through the eye-trackers as well as discomfort from the physical effects of wearing eye-trackers.

\section{Results}

The results indicated that all five instructors had challenges when determining where the cadets' visual focus were during basic and recurrent simulator sessions. Furthermore, all instructors stated that the cadets seemed to focus too much inside the ship's bridge, using ECDIS, radio, conning information and the Pilot Portable Unit (PPU). They interacted with the vessel crew at the expense of the outside view. The instructors also reported that they would like objective measurements of the cadets' eye-focus instead of the subjective data. When the cadets used eye-tracking equipment, the instructors reported that their own situational awareness increased, since they could more accurately monitor where the cadets were focusing.

The results also showed that combining simulator-based MET with eye-tracking technology could present opportunities of improvement in terms of didactics and methodology. Both instructors as well as cadets suggested that eye-tracking could be used to assess eye-focus, situational awareness as well as levels of workload and stress. One topic that emerged from the interviews was the belief that it was beneficial using eye-tracking throughout briefings and de-briefings during simulator sessions. Briefings and de-briefings could then be reviewed in groups, in solitude and with or without instructors, in real time or afterwards thus facilitating group learning or guided discoveries. About $50 \%$ of the pilots, $67 \%$ of the cadets and $67 \%$ of the instructors initially reported that their stress level and workload increased and that their situational awareness decreased when wearing eye-trackers. They stated that after spending some time wearing the eye-trackers, these effects weakened.

Eye-tracking equipment was reported to have limitations in terms of range from the master computer station for maintained visual real-time observation. The eye-tracking glasses were reported not suitable for all participants: wearing glasses (normal as well as reading glasses) and contact lenses posed an issue, since incorrect eye-focus data was generated. Participants also reported difficulties maintaining the required head posture and not gazing down below the eye-tracking glasses. 


\section{Discussion}

When striving for visual expertise, the eye-tracking methodology is considered as a useful support-tool. This is a conclusion based on previous research and confirmed in this explorative pre-study. Eye-tracking can be deployed before, during and after sessions, not only to assist the instructor with information on student eye-focus, but also for briefings. However, introducing eye-tracking equipment into existing education and training practices have some implications. These implications include technical aspects of eye-tracking equipment versus simulator installations, requiring changes in the syllabus as well as the pedagogical and didactical transformations that follow, psychological and physiological limitations of participating students, ethical issues as well as interactions with trade unions and other organizations. The pre-study furthermore showed the need for accustoming the participants into wearing eyetrackers before commencing the actual simulator session.

Knowledge in any shape can be viewed as the final form of information, which requires a substantial effort to evolve. Tacit knowledge is difficult to transfer even if methods such as socialization and externalization have been proposed. The transference of tacit knowledge is an ongoing process between the cadet and fellow senior pilots and/or instructors. Eye-tracking methodology could be a means of transferring tacit knowledge, in terms of externalization, and in order to assess and establish new standard operating procedures (SOP:s) for MET. However, in terms of socialization eye-tracking is assessed as minor effective in transferring tacit knowledge. Tacit knowledge is however transferred continuously in terms of socialization to the cadet imbedded in practical pilotage missions and in the social interaction onboard, as well as during social interaction at the pilotage station.

The results from this initial exploratory pre-study can be used as input when preparing for future studies in simulator settings with eye-glasses, including quantifiable success factors such as speed, time, navigational errors and physiological variables for example heartrate and self-assessments on stress, workload and situational awareness.

\section{Conclusions}

The exploratory pre-study indicated the following aspects:

- Eye-tracking support can be used as a mean of enhancing pilot training in simulator settings

- Eye-trackers can impose limitations on the usage of normal glasses, reading glasses and contact lenses

- Head posture, gaze behavior and wearing eye-tracking equipment in general can have implications on workload, stress levels and situational awareness

- Eye-tracking support can be useful as a didactic tool improving the situational awareness of both instructors and cadets

- Eye-tracking can be beneficial providing means of transferring tacit knowledge from pilot experts to cadets through externalization. 


\section{References}

1. Sjöfartsverket: Beskrivning av lotsyrket (2019). https://www.sjofartsverket.se/Om-oss/ Jobba-hos-oss/Om-oss/Lots/

2. Hontvedt, M.: Professional vision in simulated environments-examining professional maritime pilots' performance of work tasks in a full-mission ship simulator. Learn. Cult. Soc. Interact. 7, 71-84 (2015)

3. Haavik, T.K.: On components and relations in sociotechnical systems. J. Conting. Crisis Manag. 19(2), 99-109 (2011)

4. Crichton, M.T.: From cockpit to operating theatre to drilling rig floor: five principles for improving safety using simulator-based exercises to enhance team cognition. Cogn. Technol. Work 19(1), 73-84 (2017)

5. Sellberg, C.: Representing and enacting movement: the body as an instructional resource in a simulator-based environment. Educ. Inf. Technol. 22(5), 2311-2332 (2017)

6. Hontvedt, M., Arnseth, H.C.: On the bridge to learn: analyzing the social organization of nautical instruction in a ship simulator. Comput.-Support. Collab. Learn. 8, 89-112 (2013)

7. Sellberg, C.: From briefing, through scenario, to debriefing: the maritime instructor's work during simulator-based training. Cogn. Technol. Work 20(1), 49-62 (2018)

8. Muczyński, B., Gucma, M., Bilewski, M., Zalewski, M.: Using eye tracking data for evaluation and improvement of training process on ship's navigational bridge simulator. Sci. J. Marit. Univ. Szczec. 33(105), 75-78 (2013)

9. Karhu, K.: Expertise cycle — an advanced method for sharing expertise. J. Intellect. Cap. 3 (4), 430-446 (2002)

10. Gobet, F.: Understanding Expertise: A Multidisciplinary Approach. Palgrave, London (2015)

11. Johnson, P., Zualkernan, I., Garber, S.: Specification of expertise. Int. J. Man-Mach. Stud. 26, 161-181 (1987)

12. Dreyfus, H.L.: From socrates to expert systems: the limits and dangers of calculative rationality. In: Mitcham, C., Huning, A. (eds.) Philosophy and Technology II: Information Technology and Computers in Theory and Practice. Reidel, Boston (1985)

13. Brown, J.A.: Knowing-how: linguistics and cognitive science. Analysis 73(2), 220-227 (2013)

14. Kasarskis, P., Stehwien, J., Hickox, J., Aretz, A.: Comparison of expert and novice scan behaviors during FR flight. Paper Presented at the 11th International Symposium on Aviation Psychology, Columbus, OH (2001)

15. Polanyi, M.: Personal Knowledge. Towards a Post-Critical Philosophy. Chicago University Press, Chicago (1958)

16. Polanyi, M.: The Tacit Dimension. Anchor Books, Garden City (1967)

17. Von Krogh, G., Ichijo, K., Nonaka, I.: Enabling Knowledge Creation. Oxford University Press, New York (2000)

18. Smith, E.A.: The role of tacit and explicit knowledge in the workplace. J. Knowl. Manag. 5 (4), 311-321 (2001)

19. Nonaka, I., Takeuchi, H.: The Knowledge Creating Company: How Japanese Companies Create the Dynamics of Innovation. Oxford University Press, New York (1995)

20. Osvalder, A-L., Colmsjö, A.: Transferring tacit knowledge in process control. In: Proceedings of the 7th International Conference on Applied Human Factors and Ergonomics 2016 and the Affiliated Conferences, 29-31 July, Orlando (2016)

21. Braun, V., Clarke, V.: Using thematic analysis in psychology. Qual. Res. Psychol. 3(2), 77101 (2006) 